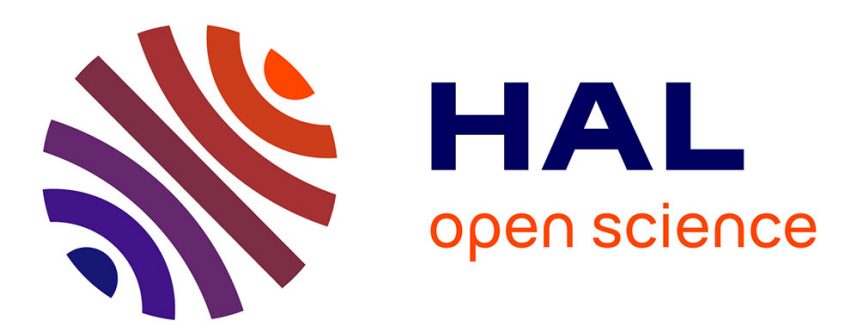

\title{
Visualisation de photographies sur une imprimante rapide d'ordinateur. Application à la simulation de topographies en pose fixe par la méthode de Lang Yves Epelboin, Alain Lifchitz
}

\section{- To cite this version:}

Yves Epelboin, Alain Lifchitz. Visualisation de photographies sur une imprimante rapide d'ordinateur. Application à la simulation de topographies en pose fixe par la méthode de Lang. Journal of Applied Crystallography, 1974, 7, pp.377-382. 10.1107/S0021889874009848 . hal-00015154

\author{
HAL Id: hal-00015154 \\ https://hal.science/hal-00015154
}

Submitted on 23 Feb 2006

HAL is a multi-disciplinary open access archive for the deposit and dissemination of scientific research documents, whether they are published or not. The documents may come from teaching and research institutions in France or abroad, or from public or private research centers.
L'archive ouverte pluridisciplinaire HAL, est destinée au dépôt et à la diffusion de documents scientifiques de niveau recherche, publiés ou non, émanant des établissements d'enseignement et de recherche français ou étrangers, des laboratoires publics ou privés. 
J. Appl. Cryst. (1974). 7, 377

\title{
Visualisation de Photographies sur une Imprimante Rapide d'Ordinateur. Application à la Simulation de Topographies en Pose Fixe par la Méthode de Lang
}

\author{
PAR Y. EPelboin et A. LifChitz \\ Laboratoire de Minéralogie Cristallographie associé au C.N.R.S., Université Paris VI, 4 place Jussieu, \\ 75230 Paris Cedex 05, France
}

(Reçu le 22 janvier 1974, accepté le 5 février 1974)

\begin{abstract}
In the simulation of X-ray section topographs by the means of a computer some problems occur concerning the representation of the photographic image. The grey scales used in the case of electron microscopy may not be applied to the case of X-ray topographs. We have studied a new grey scale and we have adapted the levels of intensity to the specific case of X-rays. Experimental and theoretical results are in good agreement but it is shown that quantitative measurements on photographic plates may be quite delicate.
\end{abstract}

\section{Introduction}

Lorsque Balibar \& Authier (1967) simulèrent, pour la première fois, l'image d'une dislocation, sur la face de sortie d'un cristal, leurs résultats durent être représentés par un dessin à la main. Après avoir retranché l'intensité du fond de la photographie, qui existe en l'absence de défaut, ils tracèrent des lignes de niveaux puis remplirent l'espace entre ces courbes avec des gris de densité variable. Outre le fait que ce procédé est extrêmement long, le dessinateur est amené à interpréter les résultats du calcul pour lever les ambiguités dues à ce procédé de courbes de niveaux.

Ces problèmes ne sont pas nouveaux. Head (1967) a mis au point, pour la représentation d'images en microscopie électronique, une échelle de teintes qui permet de simuler la photographie sur une imprimante. Ce procédé est rapide, reproductif et se prête donc bien à des études systématiques de topographies par la méthode de Lang, au moyen d'un ordinateur.

Nous avons repris cette méthode et nous l'avons utilisée pour la simulation d'images de dislocations
(Epelboin, 1974). Nous nous proposons de décrire ici l'échelle de teintes que nous avons utilisée et le problème posé par son adaptation à la réponse d'une plaque photographique aux rayons $X$. A cette occasion, nous montrerons que l'interprétation quantitative des images de topographies est extrêmement délicate, car le contraste d'une image peut varier fortement avec les conditions expérimentales.

\section{Les images de défauts en topographie aux rayons $\mathrm{X}$}

Lorsqu'on étudie un film photographique, il est usuel d'exprimer des densités de noircissement $D$ en fonction de l'éclairement du film $E$. La densité de noircissement est défini, soit en transmission (cas usuel d'un négatif), soit en réflexion (cas d'un papier photographique) par la fonction

$$
D=\log \frac{\phi_{0}}{\phi}
$$

où $\phi_{0}$ est le flux transmis (ou réfléchi) par les parties les plus blanches du film, et $\phi$ le flux reçu de la zone 
étudiée. On devrait définir $D$ comme une fonction de l'intensité du rayonnement $I$, et du temps d'exposition $t$, mais pour simplifier on préfère faire l'approximation qu'il ne dépend que du produit

$$
E=I t
$$

où $E$ est appelé l'éclairement. La forme de la courbe représentative dépend de nombreux facteurs; nous l'étudierons pour les longueurs d'ondes des rayons X.

\section{I.1 Réponse d'une plaque photographique aux rayons $X$}

Les émulsions photographiques ont un comportement variable suivant la longueur d'onde associée aux photons incidents. Dans la gamme de rayonnements qui nous intéresse, on montre (Abribat, 1953) que la densité de noircissement s'écrit:

$$
D=D_{\max }[1-\exp (-k E)] \text {. }
$$

$D_{\max }$ représente le noircissement maximum du film et $k$ est une constante caractéristique de l'émulsion employée.

Morimoto \& Uyeda (1953) ont vérifié expérimentalement cette loi dans le cas des films les plus couramment utilisés en rayons $X$.

La courbe représentative de cette fonction montre que la densité de noircissement est proportionnelle à l'éclairement lorsque celui-ci est faible mais qu'il y a assez vite saturation [Fig. 1(a)]. Mais la loi de réciprocité de Schwarzchild (1899) n'est pas toujours vérifiée et le noircissement ne dépend pas uniquement du produit $E=I t$. L'éclairement $E$ varie donc avec l'intensité reçue et le noircissement d'un film photographique dépend de la source de rayons $\mathrm{X}$ utilisée. Néanmoins, nous négligerons ce défaut qu'il est difficile d'apprécier; il est important lorsque l'éclairement est faible et tout à fait insignifiant lorsque le film est saturé.

La seconde difficulté provient d'écarts à la loi théorique (3). Si le grain du film photographique est petit, ce qui est le cas des émulsions nucléaires Ilford L4 que nous utilisons, il apparait une inertie de la couche sensible (Webbs \& Evans, 1940). Il faut, en effet, un minimum de photons pour noircir la plaque: c'est le seuil de sensibilisation. La fonction $D=f(I t)$ doit alors être représentée par la courbe $b$ de la Fig. 1. Ce défaut est important et il est nécessaire d'en tenir compte dans les interprétations d'images de topographies. Les zones les plus noires correspondent à une saturation de la plaque photographique, et les plus blanches comme l'image dynamique d'un défaut, sont en réalité sous exposées.

Une loi linéaire $D=f(I t)$ se révèle donc fausse et il devient difficile de définir une largeur d'image; nous en donnerons la preuve au paragraphe suivant.

\section{I.2 Etude expérimentale de l'influence du temps d'ex- position}

Nous avons effectué sur une même plaque photographique trois topographies en pose fixe d'un même défaut.
Les conditions de développement sont donc sensiblement les mêmes pour les trois images et seul le temps d'exposition a changé.

Sur la Fig. 2(a) on aperçoit deux dislocations courbées, dans un cristal de silicium de $800 \mu$ m d'épaisseur. Les conditions d'exposition sont classiques, le rayonnement utilisé est Mo $K \alpha$, la réflexions 220 . La puissance du tube employé est de 150 watts environ. Les deux dislocations présentent une image dynamique large, bordée de noir essentiellement sur le côté droit. Le temps d'exposition est de deux heures environ.

Sur la Fig. 2(b), il est de sept heures. L'image a changé: l'image dynamique est bordée de chaque côté

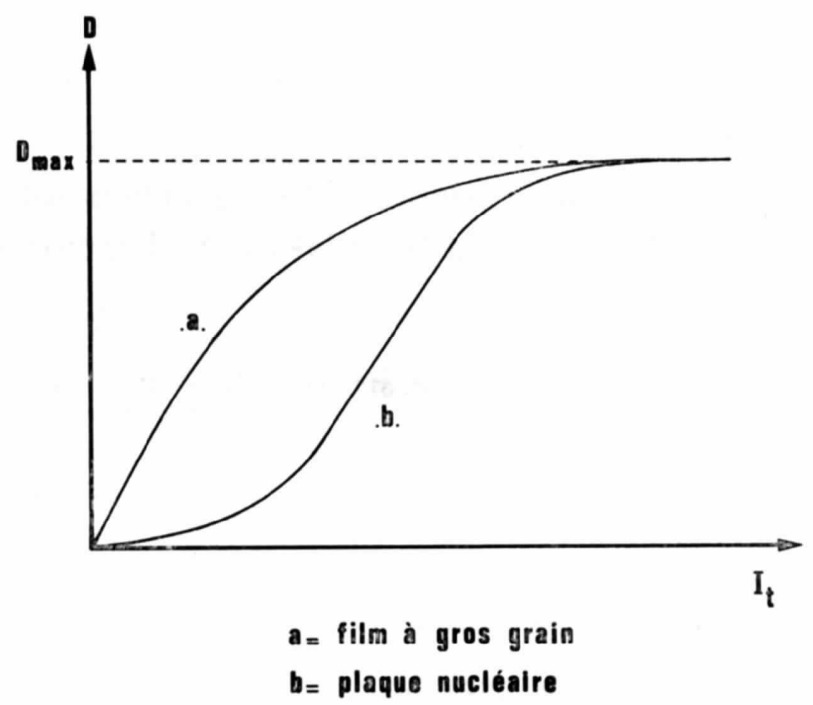

Fig. 1. Courbes de réponse en densité d'une plaque photographique aux rayons X. (a) Grain normal. (b) Plaque nucléaire à grain fin.

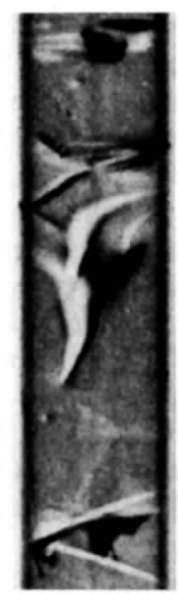

(a)

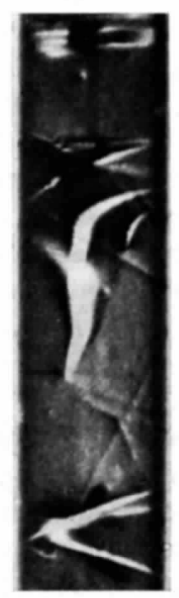

(b)

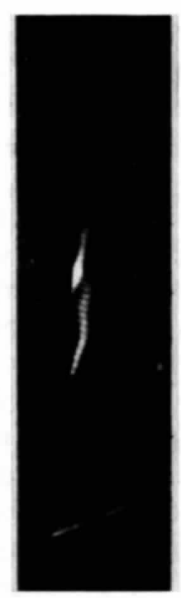

(c)
Fig. 2. Topographie dans du silicium. Réflexion 220 Mo $K \alpha$ ( $\times 39)$. (a) Exposition de 2h. (b) Exposition de 7h. (c) Exposition de $14 \mathrm{~h}$. 
de franges noires d'intensité sensiblement égales et on voit beaucoup mieux les franges blanches qui les hachurent. La largeur de l'image dynamique a diminué mais absolument pas dans le rapport des temps d'ex-

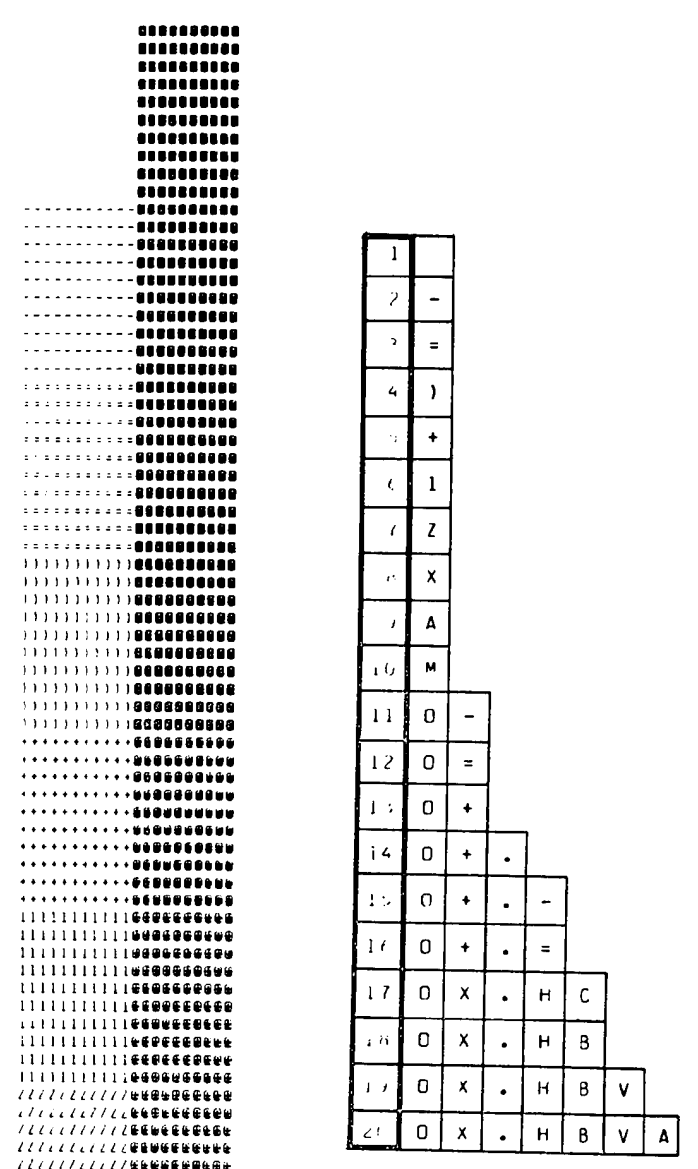

(b)

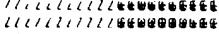

¿lilll

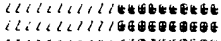

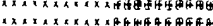

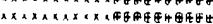

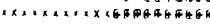

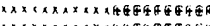

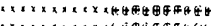

i.............

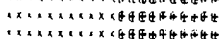

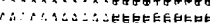

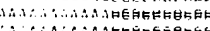

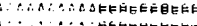

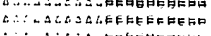

$\therefore$ A.: DA AEFGPEFPGPE

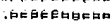

DEEEFGGEG

AFGAEGGE

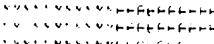

………

a.........

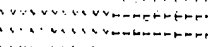

… …

(a)

Fig. 3. (a) Réduction photographique de l'échelle de teintes utilisée. (b) Combinaison des caractères nécessaires pour obtenir les teintes. Elles sont repérées, du blanc au noir par les numéros 1 à 20.

position. Tout ceci se comprend fort bien, à partir de la courbe $1(b)$. Les deux franges noires correspondent à des zones où la plaque photographique est saturée: en realité leur intensité est différente, comme le montre la Fig. 2(a); l'image dynamique correspond à des points qui sont restés en-dessous du seuil de sensibilisation, mais l'intensité du rayonnement reçu sur toute cette zone n'est certainement pas constante. On comprend qu'une mesure densitométrique, effectuée sur cette photographie, par les moyens classiques, risque d'être fort éloignée de la répartition réelle du rayonnement reçu sur la plaque. De plus, la mesure de la largeur d'image dynamique n'a qu'une valeur comparative, entre différents défauts du même cristal sur la même image mais ne peut pas être une mesure absolue. La Fig. 2(c) en est la caricature. Nous avons volontairement surexposé la plaque photographique. Tous les détails ont disparu car nous sommes dans les zones de saturation de l'émulsion et l'image dynamique révèle enfin sa structure fine: la plupart des points ont dépassé le seuil de sensibilisation, on aperçoit les franges qui la strient et sa largeur s'est considérablement amoindrie.

Cette expérience montre donc qu'un film photographique est un outil merveilleux pour apprécier qualitativement une topographie mais que son utilisation quantitative est assez délicat. Il sera nécessaire de tenir compte de toutes ces caractéristiques pour simuler une topographie à partir des résultats d'un calcul sur ordinateur qui ne peut, bien sûr, donner que des répartitions d'intensité.

\section{Les problèmes d'une échelle de teintes sur ordinateur}

Pour représenter une photographie sur une imprimante d'ordinateur, nous avons choisi la méthode de surimpression: les différents niveaux de gris sont obtenus par écriture, sur le listing, de caractères alphanumériques choisis, les teintes les plus sombres étant produites par plusieurs impressions sur la même ligne. Malheureusement, la distance entre les caractéres n'est pas la même sur une ligne ou sur une colonne, aussi la résolution spatiale de l'image diffère-t-elle dans ces deux directions. De plus, l'usure inhomogène du dispositif encreur provoque des altérations locales de l'échelle de gris. Cependant, malgré ces défauts, l'intérêt de ce procédé est qu'il est extrêmement facile à utiliser et ne fait appel à aucun périphérique spécialisé. Il est donc possible de représenter une photographie dans n'importe quel centre de calcul.

\section{II.1 Choix d'une échelle de teintes}

L'échelle de gris que nous utilisons nous a été fournie, dans sa forme initiale, par $M$ Checroun du Centre Universitaire de Dauphine à Paris. Il s'agit d'une échelle de vingt teintes que nous avons modifiée afin de corriger les anomalies qu'elle a montrées lors des mesures densitométriques. Elle est utilisable sur les imprimantes qui utilisent les caractères alphanuméri- 


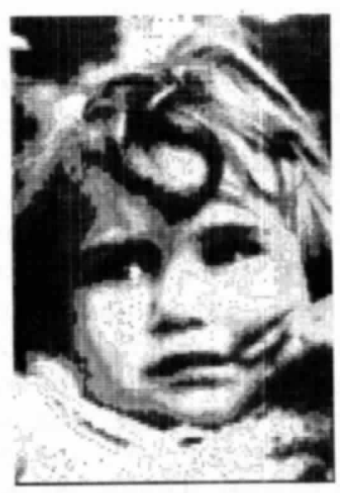

Fig. 4. Exemple de représentation photographique: Lydia.

ques standards d'IBM et comporte jusqu'à sept superpositions (Fig. 3). La Fig. 3(b) décrit les caractères nécessaires pour obtenir ces teintes. Il ne semble guère possible d'augmenter le nombre de surimpressions sans craindre des perforations accidentelles du papier. Il n'est pas souhaitable, non plus, de diminuer le nombre de passages si on désire atteindre les gris, les plus sombres possible.

A titre d'exemple, le portrait de Lydia (Fig.4) échantilloné sur une matrice $120 \times 140$, en interligne réduit de l'imprimante, montre la bonne qualité des résultats qu'il est possible d'obtenir.

II. 2 Etude de l'échelle de teintes; dynamique en lumière réfléchie et transmise

Lorsqu'on a représenté une image sur un listing, le dessin obtenu est réduit sur un banc de reproduction puis photographié sur du papier. C'est pourquoi il est nécessaire d'étudier la réponse en lumière transmise dans le cas du négatif, et en lumière réfléchie pour l'observation du positif réduit du listing.

(a) Dynamique en lumière réfléchie: Soit $I_{0}$ l'intensité lumineuse incidente; le flux réfléchi moyen correspondant à une teinte $i$ de l'échelle de gris dépend du coefficient de remplissage $T_{i}$ de la cellule élémentaire de surface $S=a b$ où $a$ est l'interligne et $b$ l'intercolonne. Si $R$ et $r$ sont les réflectances du papier vierge et du papier imprimé, respectivement, le flux réfléchi s'écrit

$$
\phi_{i}=I_{0} a b\left[R+(r-R) T_{i}\right]
$$

et si $r / R \ll 1$ comme c'est toujours le cas

$$
\phi_{i}=K\left(1-T_{i}\right) .
$$

Nous définirons la dynamique de l'échelle de teintes par la fonction $D(\phi)=\phi_{\max } / \phi_{\min }$. Cette fonction traduit l'amplitude maximale du flux que réfléchit l'échelle étudiée. Dans le cas présent:

$$
D(\phi)=\phi_{\max } / \phi_{\min }=1 /\left[1+(r / R-1) T_{\max }\right]
$$

où $T_{\max }$ représente le coefficient de remplissage de la cellule la plus noire.

Le taux de remplissage maximum $T_{\max }$ doit donc être le plus grand possible, afin d'augmenter la dyna- mique de l'échelle de teintes. Il y a donc intérêt à utiliser l'interligne le plus réduit, disponible sur l'imprimante. On retrouve aussi, ce qui est évident, que le papier doit être trés blanc et l'encre très noire.

On peut aussi définir la densité de noircissement du listing par la fonction

$$
D_{i}=\log \phi_{0} / \phi_{i}=-\log \left(1-T_{i}\right) \text { si } r / R \ll 1 .
$$

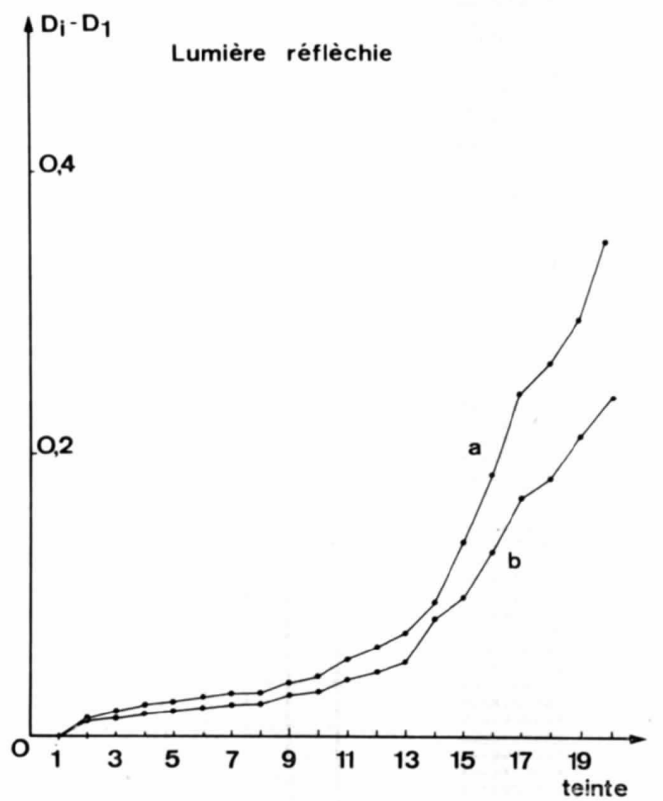

Fig. 5. Densitomètre de l'échelle de teintes par transmission, en abscisse le numéro de la teinte, en ordonnée densité de noircissement, le zéro est pris pour le papier blanc; (a) interligne $\frac{1}{8}$ pouce; $(b)$ interligne $\frac{1}{6}$ pouce.

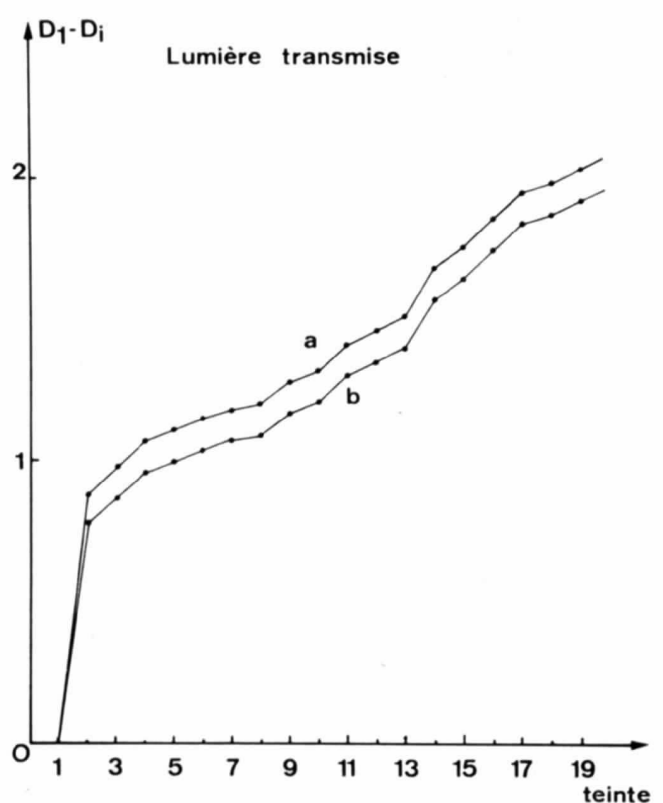

Fig. 6. Densitométrie de l'échelle de teintes par réflexion. Mêmes conventions que dans la Fig. 5. 
(b) Dynamique en lumière transmise: Cette dynamique est importante dans l'utilisation du négatif obtenu par réduction photographique du listing.

On utilise un film pour reproduction graphique, afin de conserver les caractéristiques de l'original qui ne présente que des blancs et des noirs sans gris intermédiaires. Si $t$ et $T$ sont les transmittances du noir maximum et du support du film respectivement, on montre, dans les mêmes conditions qu'auparavant que le flux transmis $\phi_{i}$ s'écrit

$$
\phi_{i}=K^{\prime} T_{i} .
$$

La cellule la plus noire, sur le listing, donne l'image la plus blanche sur le film; ceci explique que le flux transmis soit proportionnel à $T$. La dynamique de l'échelle de teintes s'écrit alors:

$$
D\left(\phi_{i}\right)=\phi_{\max } / \phi_{\min }=1+(T / t-1) T_{\max } .
$$

Elle est donc maximum si $T \rightarrow 1$ et $t \rightarrow 0$, ce qui est contradictoire: il faut optimiser le temps d'exposition de façon à noircir au maximum les zones les plus noires, sans diminuer la transparence de base du film: la densité de noircissement s'écrit

$$
D_{i}=-\log \left[t+(T-t) T_{i}\right]=-\log T_{i}+K
$$

si $1>T_{i} \gg t / T$, ce qui est toujours le cas. $K$ est une constante indépendante de la teinte qui ne dépend que de l'exposition du film.

\section{II.3 Densitomètrie de l'échelle de teintes}

Avant d'envisager l'utilisation d'une gamme de gris pour une application aussi spécialisée que la simulation de topographies aux rayons $X$, il est nécessaire de connaitre sa réponse densitométrique.

Cette étude a été effectuée sur un microdensitomètre Joyce Loebl 3CS.

Habituellement en informatique, les écritures sur

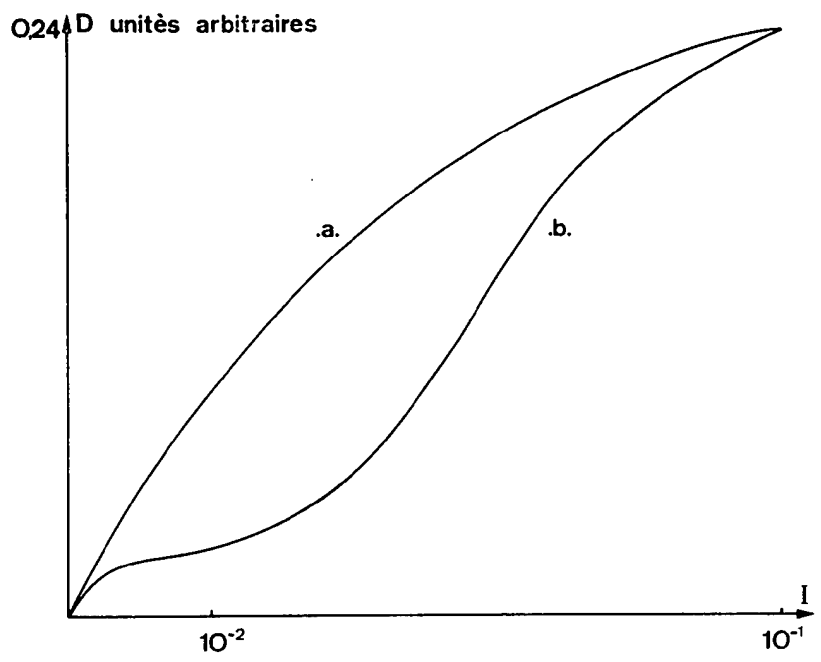

Fig. 7. Loi de réponse utilisée en simulation en topographie (a) transmission. (b) en réflexion (cas de la Fig. 8). listing se font dans la partie gauche du papier. L'usure du ruban encreur est donc la plus grande dans cette zone et l'impression d'une photographie y présente des variations de noircissement accidentelles, tout à fait aléatoires. Afin d'éliminer ce défaut, l'échelle de teintes qui a servi à nos mesures a été imprimée dans la partie droite du listing, qui correspond aux vingt dernières colonnes de l'imprimante. Les mesures sont délicates parce que le cliché est tramé. Leur principe et leur méthode sont décrits par ailleurs (Lifchitz, 1974).

Les Figs. 5 et 6 en donnent le résultat en transmission et en réflexion. La Fig. 5 montre que l'échelle est sensiblement linéaire, en transmission. Sa dynamique est la plus grande lorsque l'interligne est réduit, comme nous l'avions expliqué en établissant l'équation (5). La Fig. 6 montre, qu'en lumière réfléchie, l'échelle n'a pas une réponse linéaire. C'est là une particularité des clichés tramés. En effet, si on prend une loi de remplissage $T_{i}$, telle que l'équation (7) donne une réponse linéaire en fonction de l'intensité, alors l'équation (5) montre que l'échelle ne peut pas être linéaire en lumière réfléchie. De plus, on constate qu'il existe une discontinuité entre la première teinte, qui correspond au papier vierge, et la deuxième: le coefficient de remplissage $T_{2}$ est déjà trop élevé quoique nous ayons employé le plus petit caractère possible. Comme l'oeil intègre bien l'ensemble d'une image, ce défaut n'est guère visible dans la Fig. 4.

Lorsqu'on cherche à établir une échelle de teintes tramée, il convient donc, au départ, de se fixer le mode d'observation choisi.

\section{Adaptation de l'échelle de teintes aux topographies aux rayons $\mathbf{X}$}

Lors d'une simulation de topographie aux rayons $\mathrm{X}$, le résultat apparait sous la forme d'une répartition d'intensité sur la plaque photographique (Epelboin, 1974). Le calcul est fait dans des plans d'incidence successifs et par raison d'économie nous avons choisi un espacement qui correspond au plus grand interligne de l'imprimante. Certes, la représentation y perd en qualité, puisque la dynamique est moins bonne, mais la perte d'information est insuffisante, à notre avis, pour justifier une augmentation d'un temps de calcul déjà important. Afin de nous rapprocher, autant que possible, de la réponse représentée par la Fig. 1(b), nous avons transformé les intensités calculées par la loi suivante

$$
i=20[1-\exp (-k I)]
$$

où $i$ est le numéro de la teinte utilisée, $I$ l'intensité calculée lors de la simulation et $k$ un coefficient ajustable qui modifie la pente de la courbe de réponse et permet de saturer plus ou moins vite l'échelle de teintes.

La Fig. 7 montre la réponse densitométrique de l'échelle de teintes en fonction de l'intensité calculée. En particulier la Fig. 7(b) montre que l'on obtient avec une

JAC 7 - 5* 
assez bonne approximation la réponse théorique aux rayons $\mathrm{X}$ d'une plaque nucléaire.

A titre d'exemple, la Fig. 8 représente le résultat d'une même simulation pour deux valeurs différentes du coefficient $k$ de l'équation (8). En jouant sur la saturation des teintes, il est possible de faire apparaitre les franges de Kato qui constituent le fond de la photographie. La dislocation est faiblement inclinée dans le cristal; elle possède une image dynamique blanche, bordée de franges noires visibles uniquement sur la simulation la plus saturée. Ceci correspond assez bien à la réalité.

L'image obtenue est donc très sensible à la méthode de représentation utilisée. Auparavant, nous avions employé une échelle utilisée en microscopie électronique le cliché obtenu était à peine lisible, fort peu contrasté et nous avions le plus grand mal à analyser l'image. Cela montre que cette échelle de teintes n'est pas adaptée au cas qui nous préoccupe. Lorsqu'on veut stimuler une photographie sur une imprimante il im-

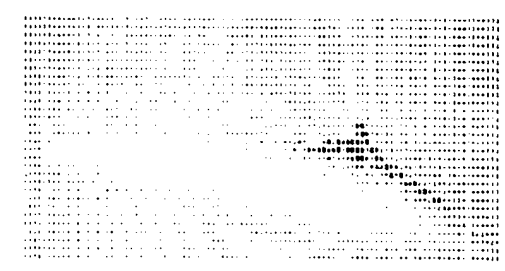

(a)

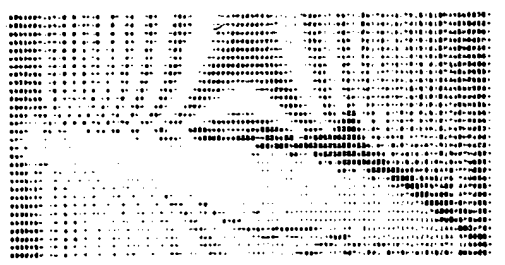

(b)

Fig. 8. Simulation d'une topographie dans le silicium. Réflexion $220 \mathrm{Ag} \mathrm{K \alpha}$. Largeur de la topographie: $180 \mu \mathrm{m}$. (a) Image pale $k=1$. (b) Image saturée $k=5$. porte donc de bien étudier auparavant la réponse des couches sensibles aux rayonnements utilisés.

\section{Conclusion}

Nous avons étudié et mis au point une échelle de teintes destinée à la représentation de photographies sur une imprimante rapide d'ordinateur. Les mesures densitométriques montrent que l'échelle de gris doit être adaptée au problème étudié.

En particulier, pour les rayons $X$, il est nécessaire de tenir compte de la réponse spécifique des couches sensibles à ces rayonnements. L'étude expérimentale confirme ce phénomène et nous avons vu que la définition d'une dimension d'image est un problème délicat.

Du fait de ses qualités. l'échelle que nous utilisons peut être utilisée pour la représentation d'autres phénomènes photographiques. Il peut être aussi intéressant de l'employer à la représentation de lignes de niveaux dans toute simulation cartographique. C'est un outil rapide, commode et peu couteux, qui ne nécessite aucun moyen particulier dans un centre de calcul. Il est possible cependant de l'améliorer par définition des niveaux de gris, mais aussi en détramant l'image. Les premiers résultats sont d'ailleurs encourageants.

Nous remercions $M$ le Professeur A. Authier et M A. Rimsky, Maitre de Recherches au C.N.R.S., pour l'intérêt et l'aide qu'ils ont apporté à ce travail.

Nous tenons à la disposition des personnes intéressées, le programme de représentation.

\section{Références}

Abribat, M. (1953). Chimie Physique des Couches Sensibles Photographiques, pp. 41-47. Paris: PUF.

Balibar, F. \& Authier, A. (1967). Phy's. Stat. Sol, 21, $413-422$.

Epelboin, Y. (1974). J. Appl. Cryst. 7, 372-377.

Head, A. K. (1967). Aust. J. Phys. 20 (5), 57-66.

Lifchitz, A. (1974). Thèse de 3ème cycle, Paris.

Morimoto, H. \& Uyeda, R. (1963). Acta Cryst. 16, $1107-$ 1119.

Schwarzchild, K. (1899). Phot. Korr. 36, 109-398.

Webb, J. H. \& Evans, C. H. (1940). Phot. J. 80, 188-196. 